\title{
ONE-YEAR RESULTS OF FIFTY CONSECUTIVE PATIENTS TREATED WITH MECHANOCHEMICAL ABLATION OF GREAT AND SMALL SAPHENOUS VEIN
}

\author{
Michal-Goran Stanisicic, ${ }^{1,2}$ Adam Węgrzynowski2,3, Katarzyna Pawlaczyk-Gabriel ${ }^{4}$ \\ 'Department of General and Vascular Surgery, Poznan University of Medical Sciences, Poznan, \\ Poland \\ ${ }^{2}$ Angiodiabetica Center for Diabetic Foot and Vascular Diseases, Poznan, Poland \\ ${ }^{3}$ Department of Diabetology, Poznan University of Medical Sciences, Poznan, Poland \\ ${ }^{4}$ Department of Hypertensiology, Angiology and Internal Diseases, Poznan University \\ of Medical Sciences, Poznan, Poland
}

\begin{abstract}
Objectives: Mechanochemical ablation (MOCA) of the great saphenous vein (GSV) and the small saphenous vein (SSV) is an alternative to thermal ablation for the treatment of superficial venous reflux. The aim of this study was to assess the efficacy of MOCA for the treatment of incompetent GSV and SSV.

Material and methods: We included 50 patients (60 legs) with incompetent GSV or SSV. Patients were aged 22-71 years, with median age of 41 years. Diameters of the saphenous veins treated were $4-16 \mathrm{~mm}$, with median diameter $9 \mathrm{~mm}$. Lengths of incompetent segments of the GSVs were $20-45 \mathrm{~cm}$, with median length $36 \mathrm{~cm}$. Lengths of incompetent segments of the SSVs were $12-25 \mathrm{~cm}$, with median length $17 \mathrm{~cm}$. We performed venous ablation using the ClariVein device with simultaneous injection of $2 \%$ polidocanol in the dose of $0.2 \mathrm{ml} \mathrm{per} \mathrm{cm}$ of the treated vein. All patients completed 12 months follow-up.

Results: In all patients the procedure resulted in complete occlusion of the incompetent segment of the saphenous vein. Additional foam sclerotherapy was needed in 41 legs (68.3\%). After 12 months partial or complete recanalisation was revealed in one GSV and 3 SSVs. The remaining veins (93.3\%) were completely occluded. During the procedure we observed transient signs of polidocanol toxicity in 2 patients.

Conclusions: MOCA using the ClariVein device is a safe method for ablation of incompetent truncal veins in patients who prefer to be managed quickly, without pain and with satisfactory results after 1 year.
\end{abstract}

Key words: ultrasound guided foam sclerotherapy, mechanochemical ablation, varicose vein surgery.

\author{
ORIGINAL PAPER \\ Phlebological Review 2015; 23, 4: 102-105 \\ DOI: $10.5114 /$ pr.2015.59018
}

Submitted: 29.11 .2015

Accepted: 12.01 .2016

\section{ADDRESS FOR CORRESPONDENCE}

Michal-Goran Stanisić

Department of General and Vascular Surgery

Poznan University of Medical Sciences

Długa 1/2, Poznań

e-mail: goranm@gazeta.pl
Mechanochemical ablation (MOCA) of the great saphenous vein (GSV) and small saphenous vein (SSV) is a relatively new method of treatment for superficial venous reflux [1-3]. This method is based on mechanical injury of the venous wall by rotating the tip of the catheter with subsequent injection of a sclerosant: polidocanol or sodium tetradecyl sulfate (STS). The method has been developed as an alternative to thermal ablation, such as endovenous laser ablation (EVLA), radiofrequency ablation or ablation with steam [4]. Recently, medical glues have provided a novel alternative to MOCA $[5,6]$. The rationale to introduce MOCA into medical practice is based on the potential risk of thermal injury of surrounding anatomic structures during EVLA or other methods of thermal ablation [4]. MOCA systems, which are cur- rently commercially available, enable ablation of the vein without the use of tumescent anaesthesia. The need for local anaesthesia is limited to the access area. The procedure can be performed on an outpatient basis, but monitoring of vital signs is recommended. MOCA requires constant ultrasound guidance. The ClariVein device (Vascular Insights, LLC, Quincy, MA, USA) is at the moment the most popular MOCA system $[1,2]$. Recently, the Flebogrif system (Balton, Warsaw, Poland) has also become commercially available [7]. Mechanochemical ablation with the ClariVein is gaining popularity among medical professionals and patients. However, since evidence concerning its efficacy is limited, at the moment MOCA is not regarded as the first-line treatment modality for varicose veins. 
This post hoc single-centre study aimed to evaluate the efficacy of MOCA using the ClariVein device for treatment of incompetent great and small saphenous veins, with a follow-up of 12 months.

\section{MATERIAL AND METHODS}

We included 50 consecutive patients (60 legs) with unilateral or bilateral incompetence of the GSV (50 veins) or SSV (10 veins). All patients were operated on by two doctors who were experienced in endovascular thermal ablation of saphenous veins and were familiar with the ClariVein system. Venous ablation using ClariVein device was performed as follows. During the procedure the patient is placed in the reverse Trendelenburg position. The vein is cannulated with a $4 \mathrm{~F}$ introducer sheath (Fig. 1). Then though this introducer sheath, under ultrasound control, the ClariVein catheter is introduced into the GSV or SSV. In a case of ablation of the GSV the tip of the catheter is positioned $2 \mathrm{~cm}$ from the saphenofemoral junction. Then the ClariVein catheter is connected to the motor drive unit. The patient's position is changed from the reverse Trendelenburg to the horizontal one and the location of the catheter tip is reassessed. With the tip of the catheter accurately placed, the motor drive unit is switched on. It rotates the wire of the ClariVein catheter with the speed of 3,500 rotations per minute. First, in order to allow spasm of the vein, the ClariVein catheter is pulled about $0.5 \mathrm{~cm}$ distally without administration of the sclerosant. Then the catheter is pulled distally with the speed of $1 \mathrm{~cm}$ per 6 seconds with simultaneous administration of a sclerosant (2\% polidocanol or $2 \%$ STS) in the dose of $0.2 \mathrm{ml}$ per $\mathrm{cm}$ of the treated vein (Fig. 2). After the MOCA procedure is completed, the patient was immediately mobilised and advised to wear class 2 compression stockings for at least two weeks.

Patients were aged 22-71 years, with median age of 41 years. According to the $\mathrm{C}$ component of the CEAP classification, 35 patients presented with $\mathrm{C} 2,10$ with $\mathrm{C} 3$ and 5 patients with $\mathrm{C} 4$ class. Diameters of the saphenous veins treated were $4-16 \mathrm{~mm}$, with median diameter $9 \mathrm{~mm}$. Lengths of incompetent segments of the GSVs were $20-45 \mathrm{~cm}$, with median length $36 \mathrm{~cm}$. Lengths of incompetent segments of the SSVs were $12-25 \mathrm{~cm}$, with median length $17 \mathrm{~cm}$. All procedures were performed using the standard technique, as above described. We used $2 \%$ polidocanol (Aethoxysklerol, Kreussler Pharma, Wiesbaden, Germany) as the sclerosant. Dosage of the drug was calculated according to the length and diameter of the incompetent vein, and adjusted to the body mass, following the dosing chart supplied by the manufacturer of the ClariVein system. Duration of use of the ClariVein catheter, which was calculated from the moment of positioning of the tip of the catheter to the moment of its withdrawal, in a case of GSV ablation was 4-10 min (median $8 \mathrm{~min}$ ). Duration of ablation of the SSV was 4-7 min (median
$6 \mathrm{~min})$. Total time of the procedure, which was calculated from the moment of puncture at the access site to the mobilisation of a patient, was 12-40 min (median 20 min). We did not perform phlebectomies, either during the primary procedure or at follow-ups. In 10 patients we performed foam sclerotherapy of side branches during the primary procedure, using $10 \mathrm{ml}$ of sclerosis foam. We also performed sclerotherapy of residual varicosities at follow-ups, which were scheduled at 4 and 8 weeks after the MOCA procedure. Additional sclerotherapies, in the case of residual varicose veins or partial recanalisation of the treated saphenous vein, were performed between 8 weeks and 6 months after the primary procedure. Additional foam sclerotherapy was needed in 41 legs and maximally 4 sessions of sclerotherapy were required. Final follow-ups were scheduled 6 and 12 months after the MOCA. We did not measure postprocedural pain with a visual analogue scale, since previous studies revealed a very low level of pain associated with this procedure [8].

\section{RESULTS}

In all patients the procedure resulted in complete occlusion of the incompetent segment of the GSV or SSV (Table 1). After 12 months partial or complete recanalisation was

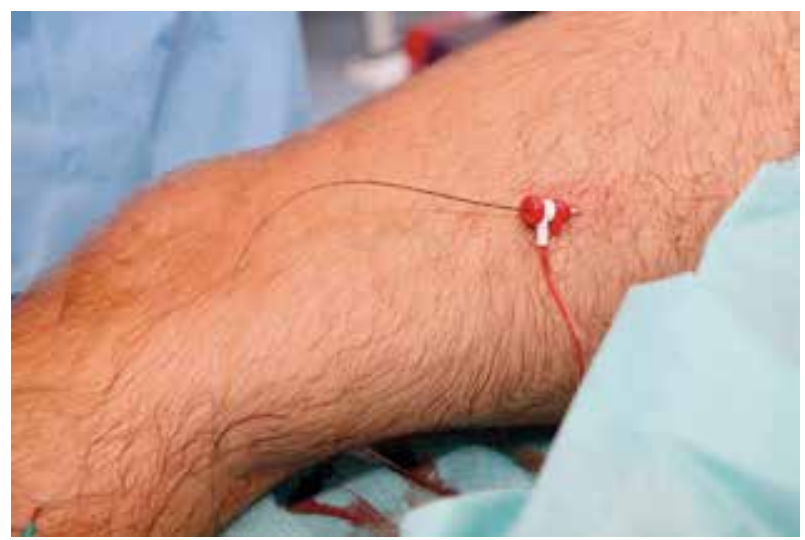

Fig. 1. Access via $4 F$ sheath introducer

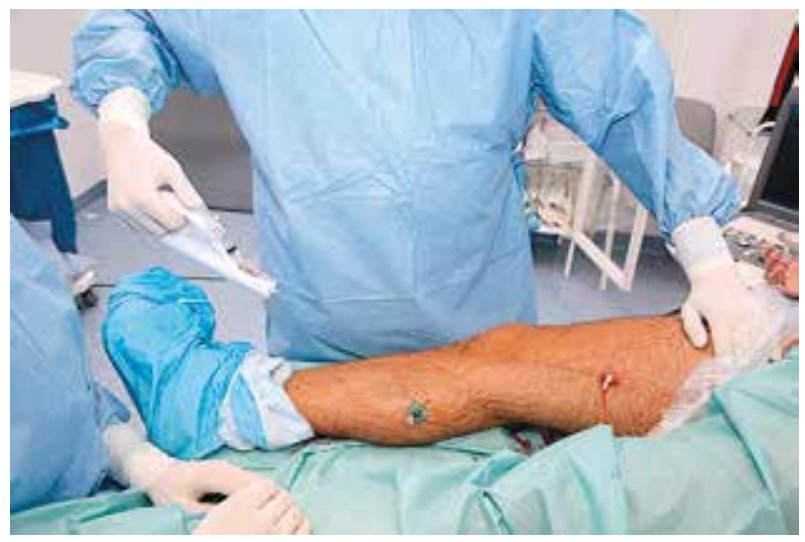

Fig. 2. With active tip rotation the catheter is retrieved with simultaneous polidocanol administration 
Table 1. Rate of GSV and SSV occlusions in follow-up

\begin{tabular}{lcccc}
\hline & \multicolumn{2}{c}{ GSV } & SSV \\
\cline { 2 - 5 } & Complete occlusion & Partial or complete recanalisation & Complete occlusion & Partial or complete recanalisation \\
\hline Technical success & 50 & 0 & 10 & 0 \\
\hline At 4 weeks & 48 & 2 & 6 & 4 \\
\hline At 8 weeks & 49 & 1 & 6 & 4 \\
\hline At 6 months & 50 & 0 & 8 & 2 \\
\hline At 12 months & 49 & 1 & 7 & 3 \\
\hline
\end{tabular}

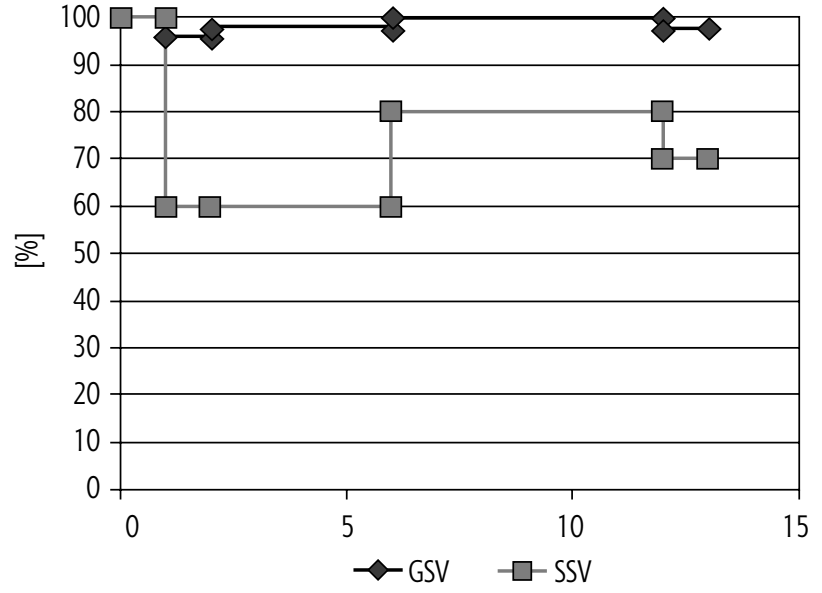

Fig. 3. Kaplan-Meier plot representing data from Table 1, including result after additional sclerotherapy

revealed in one (2\%) GSV and 3 (30\%) SSVs. The remaining $93.3 \%$ of veins were completely occluded (Fig. 3 ).

We did not observe such adverse events as deep vein thrombosis, pulmonary embolism, nerve injury or skin scarring. In 16 patients there were small, clinically insignificant haematomas at the access site or in the area of the junction of the saphenous vein with a major tributary. We observed transient signs of polidocanol toxicity, such as paraesthesia of the tongue and euphoria (comparable to a mild ethanol intoxication), which completely resolved within 10 min in 2 patients. Seven patients complained of pain at the access site, which lasted longer than 7 days. In $25 \%$ of patients we observed local indurations of the skin at the access site or in the areas of varicosities managed with foam sclerotherapy.

\section{DISCUSSION}

According to the European Society for Vascular Surgery 2015 guidelines, thermal ablation is the treatment of choice for incompetent GSV and potentially for the management of incompetent SSV [4]. Other endovascular methods, such as MOCA or the use of medical glues (for example, Venaseal, Medtronic, Minneapolis, MN, USA) are mentioned as promising methods, but with insufficient evidence of long-term efficacy [4-6].
The development of non-thermal minimally invasive methods for ablation of the GSV and SSV was aimed at reducing the risk of adverse events and invasiveness of the procedure. In addition, tumescent anaesthesia is poorly tolerated by some patients, while the risk of injury to the saphenous or sural nerves is quite high during below-the-knee thermal ablations [6, 8]. After minimally invasive surgical procedures the patient should be able to go back to work and be fully active during everyday activities (such as driving a car). Patients after thermal ablation, regardless of the method used, often complain of bruising, oedema and prolonged swelling related to the procedure (thermal injury and/or tumescence) [4-6, $9,10]$. From the point of view of a medical professional the cost of purchase and maintenance of the device is important. On the other hand, a patient is primarily interested in a procedure that can be performed on an outpatient basis, with a minimal volume of anaesthetic fluid used, which will enable a return to work immediately after the treatment. The ClariVein is a disposable device, with no need for purchase of additional equipment. The patient can be fully mobilised immediately after completion of the procedure. Since there is no thermal injury and no tumescence anaesthesia, the risk of oedema related to the treatment is minimised.

It should be mentioned that our patients did not opt for an open surgical intervention for varicose veins (such as crossectomy or miniphlebectomy) and also did not accept tumescent anaesthesia. Before consenting to the MOCA, each patient was given an opportunity to be managed using a standard thermal ablation procedure (EVLA or RF ablation). Patients accepted the scenario in which an additional foam sclerotherapy would be needed in order to close incompetent tributaries. They were also were advised that follow-up visits would be necessary to achieve a good final result. It should be emphasised that for the time being endovascular treatments for varicose veins are not reimbursed by the Polish health care system and the patients participating in this study have paid for the procedure, including sclerotherapies performed at follow-ups.

Another important issue concerning the ClariVein system is the efficient and safe dose of the sclerosant. Early studies on this system used $1.5 \%$ polidocanol or 
STS, achieving 1-year closure rates of the GSV and SSV of $90-92 \%$ [1-3]. Such an $8-10 \%$ rate of recanalisation, if compared to the results of thermal ablation procedures, does not seem satisfactory. Later studies used $2 \%$ polidocanol, and such a concentration of sclerosant is currently recommended by the manufacturer of the device. With this concentration of sclerosant 1-year closure rates were higher: $94-96 \%[1-3,5,6,9,10]$. However, it has been observed that if the first $10 \mathrm{~cm}$ of the GSV were managed with $3 \%$ polidocanol, the results could be even better [11]. Such a dose is not currently advised by the manufacturer of the ClariVein, but a recently published report by Lam et al. suggests that such a modification may be justified [12]. Presented data opt against use of foam in GSV and SSV [12]. In our SSV patients we observed quite a high recurrence rate, but these recurrences were probably primarily related to anatomical factors - in all cases the diameter of the saphenous vein was more than $15 \mathrm{~mm}$. Concomitant medications could also play a role: one of these patients, presenting with atrial fibrillation, was anticoagulated with rivaroxaban after the procedure.

Contrary to thermal methods of ablation, the ClariVein system requires an additional foam sclerotherapy to achieve a good final cosmetic result. In our patients all hyperpigmentations were related to foam sclerotherapy of the tributaries. Patients should be informed about possible hyperpigmentation resulting from foam sclerotherapy. Another disadvantage of the ClariVein system is the fact that simultaneous management of bilateral varicosities can be associated with the administration of too high dose of the sclerosant. Thus, usually varicose veins of only one leg can be treated, which significantly increases the total cost of treatment in patients with bilateral disease. In this study we managed only varicose vein patients presenting with class $\mathrm{C} 2$ to $\mathrm{C} 4$, yet the ClariVein system can also be used in patients with open and healed venous ulcers, since the risk of infection in such patients is low if a MOCA method is used. Also, since only a minimal dose of local anaesthetic is required, the ClariVein can be used in elderly patients. In our opinion, MOCA is a good alternative to thermal methods of ablation.

\section{CONCLUSIONS}

MOCA using the ClariVein device is a safe method for ablation of incompetent truncal veins in patients who prefer to be managed quickly, without pain and with satisfactory results after 1 year. Concentration of the sclerosant used for ablation of the first $10 \mathrm{~cm}$ of the saphenous vein should be established by future trials.

The authors declare no conflict of interest.

\section{References}

1. van Eekeren R.R., Boersma D., Elias S., Holewijn S., Werson D.A., de Vries J.P., Reijnen M.M. Endovenous mechanochemical abla- tion of great saphenous vein incompetence using the ClariVein device: a safety study. J Endovasc Ther 2011; 18: 328-334.

2. Elias S., Raines J.K. Mechanochemical tumescentless endovenous ablation: final results of the initial clinical trial. Phlebology 2012; 27: 67-72.

3. Mueller R.L., Raines J.K. ClariVein mechanochemical ablation: background and procedural details. Vasc Endovascular Surg 2013; 47: 195-206

4. Wittens C., Davies A.H., Bækgaard N., Broholm R., Cavezzi A., Chastanet S., de Wolf M., Eggen C., Giannoukas A., Gohel M., Kakkos S., Lawson J., Noppeney T., Onida S., Pittaluga P., Thomis S., Toonder I., Vuylsteke M., Kolh P., de Borst G.J., Chakfé N., Debus S., Hinchliffe R., Koncar I., Lindholt J., de Ceniga M.V., Vermassen F., Verzini F., De Maeseneer M.G., Blomgren L., Hartung O., Kalodiki E., Korten E., Lugli M., Naylor R., Nicolini P., Rosales A.; European Society for Vascular Surgery. Editor's Choice - Management of Chronic Venous Disease: Clinical Practice Guidelines of the European Society for Vascular Surgery (ESVS). Eur J Vasc Endovasc Surg 2015; 49: 678-737.

5. Morrison N., Gibson K., McEnroe S., Goldman M., King T., Weiss R., Cher D., Jones A. Randomized trial comparing cyanoacrylate embolization and radiofrequency ablation for incompetent great saphenous veins (VeClose). J Vasc Surg 2015; 61: 985-94.

6. Lawson J., Gauw S., van Vlijmen C., Pronk P., Gaastra M., Mooij M., Wittens C.H. Sapheon: the solution? Phlebology 2013; 28 Suppl 1: 2-9.

7. Ciostek P., Kowalski M., Woźniak W., Miłek T., Myrcha P., Migda B. Phlebogriffe - new device for mechanochemical ablation of incompetent saphenous veins: a pilot study. Phlebol Rev 2015; 23: 72-77.

8. van Eekeren R.R., Boersma D., Konijn V., de Vries J.P., Reijnen M.M. Postoperative pain and early quality of life after radiofrequency ablation and mechanochemical endovenous ablation of incompetent great saphenous veins. J Vasc Surg 2013; 57: 445-450.

9. Bishawi M., Bernstein R., Boter M., Draughn D., Gould C.F., Hamilton C., Koziarski J. Mechanochemical ablation in patients with chronic venous disease: a prospective multicenter report. Phlebology 2014; 29: 397-400.

10. Boersma D., van Eekeren R.R., Werson D.A., van der Waal R.I., Reijnen M.M., de Vries J.P. Mechanochemical endovenous ablation of small saphenous vein insufficiency using the ClariVein $\left({ }^{\circledR}\right)$ device: one-year results of a prospective series. Eur J Vasc Endovasc Surg 2013; 45: 299-303.

11. Elias S., Lam Y.L., Wittens C.H. Mechanochemical ablation: status and results. Phlebology 2013; 28 Suppl 1: 10-14.

12. Lam Y.L., Toonder I.M., Wittens C.H. Clarivein ${ }^{\star}$ mechanochemical ablation an interim analysis of a randomized controlled trial dose-finding study. Phlebology 2015 Aug 6. pii: 0268355515599692. [Epub ahead of print]

13. Moore H.M., Lane T.R., Franklin I.J., Davies A.H. Retrograde mechanochemical ablation of the small saphenous vein for the treatment of a venous ulcer. Vascular 2014; 22: 375-377. 